\title{
Le débat francophone
}

\section{Cyrille François}

\section{OpenEdition}

\section{Journals}

Édition électronique

URL : http://journals.openedition.org/recherchestravaux/413

DOI : 10.4000/recherchestravaux.413

ISSN : 1969-6434

Éditeur

UGA Éditions/Université Grenoble Alpes

\section{Édition imprimée}

Date de publication : 30 juillet 2010

Pagination : 131-147

ISBN : $978-2-84310-174-8$

ISSN : 0151-1874

Référence électronique

Cyrille François, «Le débat francophone », Recherches \& Travaux [En ligne], 76 | 2010, mis en ligne le 30 janvier 2012, consulté le 08 septembre 2020. URL : http://journals.openedition.org/recherchestravaux/ 413 ; DOI : https://doi.org/10.4000/recherchestravaux.413 
Cyrille François

Université de Cergy-Pontoise

CRTF EA 1392

\section{Le débat francophone}

Pour le lecteur ordinaire, le syntagme "littérature francophone» semble aller de soi. Or, voilà deux décennies que la francophonie et la francophonie littéraire sont mises en débat - et les remous s'apaisent difficilement, en relation à la politique, l'Histoire, la culturer. En ligne de mire : la terminologie. L'épithète "francophone» est comme un tissu malmené que l'on déchire, distend, rétrécit... Ce n'est pas qu'une querelle d'érudits pointilleux : les écrivains s'y mêlent régulièrement, avec un ton plus assuré et à grand renfort de propos généraux. Face à cet imbroglio, il s'agira dans ces pages, non de trancher le débat ou de prétendre en clarifier tous les tenants et aboutissants, mais au moins d'en poser quelques termes de façon synthétique. Mon point de départ sera le récent et polémique manifeste Pour une littérature-monde 2 . Dans un premier temps, comme dans les deux contributions précédentes, j'esquisserai un panorama des grandes tendances afin d'asseoir, ensuite, des propositions qui ne se voudront ni péremptoires, ni définitives : l'interrogation restera et doit rester ouverte.

I. Afin de défaire au plus tôt quelques nœuds et éviter quelques chausse-trappes, précisons d'emblée qu'il sera question ici de francophonie littéraire, non de francophonie politique ou linguistique : quoique liées, elle ne se recouvrent pas. Cette distinction est de taille : elle entre pour une bonne part dans le débat. La francophonie littéraire regroupe toute production littéraire en langue française. Traditionnellement, on en retranche la littérature française, soustraction qui fait justement débat.

2. M. Le Bris, J. Rouaud, Pour une littérature-monde, Gallimard, 2007. 


\section{Français ou francophonie ? Le débat}

\section{Les termes du débat}

La parution en 2006 du manifeste Pour une littérature-monde, dont nombre de signataires, autour de Jean Rouaud et Michel Le Bris, disposent d'une reconnaissance dans le champ littéraire français, prétendait apporter une solution au débat qui agitait depuis plusieurs années déjà le milieu universitaire. Ce débat, nul ne l'ignore désormais, oppose ceux qui maintiennent la différence entre littérature française et littérature francophone à ceux qui y voient une perpétuation de la politique culturelle colonialiste et préconisent en conséquence de tout rejeter en bloc pour empêcher de penser toute différence.

Une première position dans ce débat pose l'équivalence entre littérature de langue française et littérature française. C'est la thèse d'Anne-Rosine Delbart, qui prône l' "addition de la littérature francophone à la littérature française $e^{\text {" }}$ plutôt que l'inverse. Ce point de vue est aussi celui d'André Brincourt dans son essai Langue française terre d'accueil ${ }^{4}$ ou encore, pour des raisons différentes, de l'écrivain Nimrod, lorsqu'il déclare :

Comment peut-on être francophone ? Justement, on ne le peut. [...] Senghor l'appellera "francophonie», c'est-à-dire une démonstration des qualités propres à la francité. Quant aux écrivains dits francophones, ce sont des Français à part entière, d'où qu'ils viennent's.

Une telle opération est difficilement tenable dans la mesure où elle entretient une confusion entre langue et identité. Certes, ce renversement des discours habituels sur le rapport français/francophone s'accompagne d'une précision de taille :

Autrement dit, c'est la littérature française cantonnée à un pays ou à une région qui demandera une définition complémentaire, non la littérature française en général $[\ldots]^{6}$.

Qu'à cela ne tienne : l'impasse n'est pas loin. Il n'existerait pas de littérature québécoise mais une littérature française du Québec...7 ainsi qu’une

3. A.-R. Delbart, Les Exilés du langage : un siècle d'écrivains français venus d'ailleurs : IgIg2000, Pulim, Limoges, 2005, p. 13.

4. A. Brincourt, Langue française terre d'accueil, Éditions du Rocher, 1997. Que ce soit chez Brincourt ou Delbart, le propos n’illustre pas pour autant un discours dépréciatif à l'encontre des auteurs choisis, au contraire.

5. Nimrod, «Le nouveau monde», Magazine littéraire, $\mathrm{n}^{\circ}$ 45I, mars 2006, p. 46-47.

6. A.-R. Delbart, op. cit., p. I4.

7. La Révolution tranquille, avec la génération de L'hexagone, fonde précisément une littérature qui se veut québécoise, bien dissociée de la française, à la suite d'autres moments charnières, comme la querelle Crémazie-Casgrain ou l'École littéraire de Montréal. 
littérature française de France. Pour ceux qui traquent le néo-impérialisme, une formulation de ce type serait inadmissible car elle ne ferait rien d'autre que renforcer le lien de dépendance à la littérature française. Au demeurant, quitte à redéfinir une catégorie, on voit mal pourquoi ce serait la littérature française et non la littérature francophone, puisque cela reviendrait au même. C'est pourquoi poser un premier garde-fou - en passant sur les imprécisions que cela peut véhiculer - nous permettrait de parer d'ores et déjà à quelques contresens : la littérature française est de langue et de culture françaises.

Quelles seraient les autres possibilités ? inverser l'ordre ? Dans ce cas, la littérature française serait une littérature francophone comme les autres, car, réduit à sa signification la plus élémentaire, le syntagme «littérature francophone» reformule simplement «littératures de langue française». La littérature francophone arbore par là même les traits de sa dépolitisation, de sa prise d'indépendance à l'égard de l'idéologie attachée à la pureté linguistique française. On s'aperçoit alors qu'en intégrant la littérature française aux littératures francophones, on échappe au paternalisme culturel, à la supériorité orgueilleuse d'une littérature française encline à la discrimination. Alain Mabanckou, dans le manifeste, synthétise cette position :

[...] on a pu remarquer le flou que véhiculait la notion de francophonie, non pas que celle-ci soit à décrier mais par l'allusion fort politique qu'elle sous-tend, et jamais une notion n'avait été aussi contestée, les procureurs les plus impitoyables regardant la francophonie comme la continuation de la politique étrangère de la France dans ses anciennes colonies! La création littéraire est étrangère à ces rapports, et c'est dans cet esprit que je suggérai alors la définition de ce qu'il fallait entendre par "écrivain francophone», définition dans laquelle j’englobais également, sans tergiversations, l'écrivain français $[\ldots]^{8}$.

Ces propos appellent deux remarques : premièrement, Alain Mabanckou reconduit l'amalgame entre francophonie politique et littérature francophone qui sert de support à la revendication d'une littérature-monde, oubliant de la sorte que l'une et l'autre, bien que fortement liées, sont distinctes. On constate ensuite que le débat se résume à un problème terminologique et, en particulier, connotatif.

Toujours est-il que, malgré tout, l'addition de la littérature française à la littérature francophone serait une meilleure option que l'inverse. Admettons donc, au moins provisoirement, l'intégration de la littérature française dans la littérature francophone9. En apparence, les conflits sont réglés; seuls

8. A. Mabanckou, "Le chant de l'oiseau migrateur», Pour une littérature-monde, op. cit., p. 55-56.

9. Parmi ceux qui soutiennent avec force ce point de vue, on pourrait citer M. Fernandes dans son dernier ouvrage : Les Écrivaines francophones en liberté : écriture de l'hybridité postcoloniale et métaphores cognitives, L'Harmattan, 2007. 
demeurent insatisfaits les réfractaires cramponnés sur les positions d'arrièregarde de la domination. Deux objections interviennent alors.

La première persiste à récuser une telle dénomination. C'est le cas des adversaires de la littérature francophone : à l'instar des indécis, ils tentent souvent d'esquiver le problème en substituant à l'ensemble «littérature francophone" une autre terminologie plus satisfaisante. Il y a pour eux comme une urgence à vouloir coûte que coûte effacer toute trace de la «francophonie littéraire», afin d'en finir avec son fardeau de présupposés. En fustigeant l'idéologie inhérente à la francophonie, ils s'en prennent - à raison - aux relents du colonialisme, à sa perpétuation dans l'impérialisme culturel, à la hiérarchie qui met au second plan les littératures dites "francophones», ou, encore à la lecture ethnocentriste des textes ${ }^{\mathrm{I}}$. À leurs yeux, la francophonie reste captive de l'histoire. D'une certaine manière, ils pénalisent la langue pour ce dont elle n'est pas coupable, comme le souligne judicieusement Daniel Maximin dans sa Géopoétique de la Carä̈be :

On sait bien que les modes, les genres, les styles, les langues n'ont pas de pureté originelle, ni de prédestination à libérer ou à emprisonner. La langue-carcan du Code noir ne saurait se confondre avec celle qui, de Montaigne à l'abbé Grégoire et Hugo, jusqu’à Roumain, Damas, Césaire ou Fanon, a balisé aussi les chemins décolonisés de la libertér ${ }^{\text {In }}$.

Cette confusion entre francophonies institutionnelle et littéraire signalée plus haut pousse nombre d'écrivains francophones comme Nimrod - c'està-dire de langue française -à refuser cet adjectif. Dans la même optique, le manifeste Pour une littérature-monde, emblématique des désaveux jusqu'ici émis à l'encontre de la littérature francophone, propose lui aussi d'en finir avec la francophonie.

\section{Pour une littérature-monde en français ?}

Dans la lignée du festival «Étonnants Voyageurs", le manifeste rassemble des écrivains et intellectuels, français ou exilés de leur pays d'origine, qui refusent l'appellation de «littérature francophone». Ils lui substituent une «littératuremonde en français» dont l'intérêt est double : jeter aux oubliettes la francophonie littéraire et renouer avec le geste référentiel de la littérature contre la prétendue autarcie du texte. Désormais libéré de toute appartenance, l'écrivain de langue

IO. Lecture selon laquelle les textes se réduisent à des documents illustrant les mœurs de telle ou telle peuplade (anciennement) colonisée

II. D. Maximin, Les fruits du cyclone : une géopoétique de la Caraïbe, Gallimard, 2006, p. 3 I. 
française explore le monde pour le dire, traversant les frontières géopolitiques, se mettant au service du roman comme genre total, métisse, plurilingue.

Plusieurs défauts écornent la revendication du manifeste. En premier lieu, son militantisme revigorant abuse de quelques généralisations malvenues. Il pèche souvent par son manque de rigueur scientifique, à commencer par l'absence de définition de la francophonie, ce qui, il faut l'avouer, entraîne toujours les mêmes raccourcis et ambiguïtés. En outre, les auteurs ont tendance à ne pas s'accorder sur la signification de la littérature-monde; de telles divergences, bien qu'elles profitent au débat, témoignent du flou terminologique, de son invalidité indépendamment du débat actuel. Tandis que le manifeste paraît accorder une visée globale à la littérature-monde, tout en lui assignant une "poétique» et un imaginaire spécifiques, celui d'une écriture épousant le bruissement, les rythmes du monde, Michel Le Bris, dans son article très exalté en ouverture du volume, semble réduire le champ envisagé aux écrivains exilés ou plus généralement à la croisée des cultures. De ce groupe, pourtant loin de constituer un échantillon représentatif de la francophonie littéraire, il fait l'ambassadeur de la littérature de demain, sans attaches, vagabonde, «à cheval entre plusieurs cultures ${ }^{12}$ » :

Littérature-monde, très simplement pour revenir à une idée plus large, plus forte de la littérature, retrouvant son ambition de dire le monde, de donner un sens à l'existence, d'interroger l'humaine condition, de reconduire chacun au plus secret de lui-même. Littérature-monde pour dire le télescopage, dans le creuset des mégapoles modernes, de cultures multiples, et l'enfantement d'un monde nouveau. Littérature-monde, enfin, à l'heure où sur un tronc désormais commun se multiplient les hybridations, dessinant la carte d'un monde poly phonique, sans plus de centre, devenu rond $[\ldots]^{13}$

Michel Le Bris tend ici à mélanger plusieurs phénomènes propres à l'internationalisation - ou mondialisation - du monde, certes concomitants, mais n'intervenant pas aux mêmes niveaux, ni problématiques de la même façon : l'écriture en présence de toutes les langues, les références intertextuelles puisées dans la littérature mondiale, la littérature viatique, la littérature «nationale» se métissant... Alain Mabanckou, quant à lui, étend la littératuremonde à toute œuvre écrite en français; Nimrod et Abdourahman Waberi font de même. Ils la dépeignent comme :

I2. M. Le Bris, "Pour une littérature-monde en français», Pour une littérature-monde, op. cit., p. 35 .

I3. Ibid., p. 4I-42. L'empreinte d'Édouard Glissant y est flagrante, de sa poétique du Divers à son Traité du Tout-monde. L'idéalisation et la généralisation sont les mêmes. 
[...] le concert de la multiplicité d'expériences, la reconnaissance de la force de l'art dans ce qui apparaît comme le "désordre de la vie». Elle part du constat qu'il nous faut désormais imaginer l'écrivain dans sa mobilité et dans l'influence que suscite en lui l'émerveillement de ce qui ne vient pas nécessairement de son univers ${ }^{14}$.

Finalement, la littérature-monde remplace une notion générale - la littérature francophone - par une autre tout aussi générale, sans se donner véritablement les moyens de dire la diversité des francophonies dans la mesure où elle se focalise surtout sur la déterritorialisation, l'hybridité, le nomadisme. La littérature-monde s'ajoute donc à une liste toujours jugée insatisfaisante : francophonie, francographies, francopolyphonies... tout en lui ménageant une spécificité interculturelle dans sa déterritorialisation.

Loin de moi l'envie de clouer ce manifeste au pilori : on peut savoir gré aux signataires de rétablir quelques vérités, notamment sur le purisme francocentriste. Leurs opinions variées stimulent la réflexion, et il y a une urgence à ne pas se taire devant le rejet du plurilinguisme, l'amputation des richesses culturelles, le cloisonnement pour assurer les replis... La présence des langues et des cultures, assène Boualem Sansal, est une chance que l'on piétine trop volontiers, lorsque le français traite de haut les autres ou lorsque les jeux de pouvoirs étouffent la vivacité et la permanence de cette langue. Que ce soit pour elle ou pour les autres, l'ambition de la littérature - des littératures-mondes selon Lyonel Trouillot - est d'aider à préserver la diversité des langues, des réalités, des "êtres-aux-mondes", pour que l'universalité ne se trompe pas de lendemains. Enfin, on ne peut que se féliciter de la visibilité dont bénéficie cette entreprise dans le champ littéraire français, force que n'aurait pas eue un ouvrage universitaire ${ }^{15}$.

\section{La dialectique français/francophone}

En cherchant une dénomination commune, les propositions du débat se fondent sur les exemples d'écrivains très contemporains, qui se désintéressent de la perspective historique. C'est cette dynamique que veulent maintenir ceux qui conservent la distinction entre littérature française et littérature francophone. Selon les tenants de cette thèse, la centralité a existé, existe toujours. Les littératures francophones ont pour origine, à quelques exceptions près très

I4. A. Mabanckou, op. cit., p. 65.

15. C'est aussi ce qui peut le décrédibiliser, car comme pour tous les écrivains francophones reconnus, le rejet du Centre franco-parisien s'accommode pourtant d'une publication dans les grandes écuries de ce centre. 
localisées, une rupture, une inadéquation entre langue et culture ${ }^{16}:$ la langue a été emportée dans les bagages des immigrants (Québec, Acadie), s'est étendue sur des territoires à la culture plus ou moins différente de la centralité française (régions de France, Suisse, Belgique, Roumanie...), s'est dispersée en pointillés ou a été imposée (anciennes colonies, DOM-TOM). Pour toute l'époque des indépendances et du postcolonialisme, l'écriture francophone se repère par sa réappropriation subversive d'une langue, de représentations, de codes génériques dans lesquels elle marronne pour élaborer par dérivation une nouvelle identité culturelle ainsi qu'une nouvelle littérature ${ }^{17}$.

Cet axe du décentrement, qui existe autant hors de France qu'en France (notamment avec les littératures des immigrations), est remis en cause aujourd'hui parce qu'il n'explique plus tous les faits littéraires francophones en raison de l'autonomisation des littératures. Par ailleurs, le fait que beaucoup d'écrivains actuels ne perçoivent pas la langue française comme une langue étrangère, mais rapprochent leur pratique littéraire de celle de n'importe quel écrivain, rend caduque cette dualité. Enfin, le cloisonnement des champs ainsi que l'importance de nouveaux centres éditoriaux et culturels comme Montréal rompt parfois la dépendance.

Celle-ci conserve néanmoins une pertinence historique. La pratique littéraire du détour ou, dans le cadre de la littérature coloniale, de l'imitation, a caractérisé les littératures francophones hors de France dans une tension positive ou négative liant littérature française et auteurs "autochtones». Les interactions entre centre et périphérie, français et francophone correspondent donc à des relations d'imitation ou de répulsion, de réappropriations ou de transformations de codes. Le fait littéraire francophone repose sur une dynamique historique, pour ne pas dire une dialectique, entre la langue française en France et hors de France, de même qu'il existe une tension entre langue française et langues régionales ou étrangères en France.

Cette distinction n'ordonne pas une hiérarchie, elle n'oppose pas deux blocs : la pluralité est partout, dans la littérature française elle-même, parcourue en synchronie et en diachronie par le plurilinguisme, par la mobilité

I6. Cette notion de "culture" pose problème à quelques égards, tout comme celle d'ethnie par exemple : elle est fluctuante, sujette à la porosité, entraînée dans l'hybridité croissante du monde. C'est pourquoi, il semble aujourd'hui de plus en plus difficile de parler de «socle culturel». On peut pourtant parler d'une congruence de traits culturels composant une source, la singularité d'une «culture».

17. On objectera que c'est le cas pour tout(e) écrivain(e). Mais d'une part une telle génération semble loin d'être avérée (à moins qu'elle ne se pose en règle excluant tous ceux qui ne la suivent pas); d'autre part, la situation de l'écrivain francophone ne s'évalue pas à l'intérieur d'une société donnée de laquelle il serait décroché. Elle engage un dialogue ou des querelles de culture. Elle est supra-sociétale. 
des formes, par l'évolution des systèmes. Cette distinction est située historiquement, ce qui implique qu'elle n'aura peut-être plus cours à l'avenir du fait de l'autonomisation de toutes les littératures, ou de leur confusion complète. En tant que telle, elle ne s'oppose pas à l'annulation de la distinction : elle constitue une mise en relief du continuum littérature française/francophone, ou une posture volontariste d'analyse des textes francophones ${ }^{18}$.

\section{Un paradigme francophone?}

\section{De la forme au fond du problème}

À bien des égards ce débat, bien que vivifiant, se fourvoie parfois par excès de passions, par manque de rigueur, par confusion entre francophonie littéraire et francophonie institutionnelle. On y reconnaît surtout la croyance un peu naïve selon laquelle changer le mot éradique les maux. Or, ce n'est pas tant le terme qui doit être accusé que la chose qu'on l'a amené à désigner. Si l'on pouvait réduire la question au changement d'étiquette, la difficulté serait vite résolue. Hélas, la détermination linguistique ne peut suffire ${ }^{19}$.

Les différentes positions évoquées ci-dessus ne sont sans doute pas incompatibles. De cet aperçu sommaire et stéréotypé, nous pourrions conclure provisoirement sur la polysémie de la francophonie littéraire, polysémie qui débroussaille le débat sans le clore. On distinguerait alors :

- une acception linguistique où francophone signifie : "d'expression française»;

- une acception poético-historique ou heuristique : participant d'une mise en perspective historique de certains corpus, elle préserve la différence entre littérature française et littérature francophone.

Ces deux acceptions portent en dernière analyse sur des questions de forme. Dès que l'on se penche sur le fond, beaucoup reste à penser. Car derrière l'illusion d'un ensemble francophone grouille une diversité de situations (socioculturelles) d'énonciation, de pratiques littéraires; il n'est pas jusqu'à la langue qui ne soit sous le coup de cette diversité puisque l'homogénéité $\mathrm{du}$ français n'existe pas dans les faits. La norme elle aussi, plus abstraite que concrète, connaît des contrepoids : le français est devenu une langue polycentrée. Il n'existe pas de langue pure, idéale, intacte, essentiellement liée à

18. En France, il s'agit aussi d'une construction pédagogique réactive au contexte culturel, permettant de valoriser des textes en mal de reconnaissance.

19. La citation d'Alain Mabanckou suggérait bien la nécessité de veiller plus au signifié qu'au signifiant. 
l'universalité, ni une seule norme. C'est pourquoi le pluriel est probablement plus pertinent lorsque l'on parle de littérature(s) francophone(s).

La querelle terminologique laisse donc échapper un problème plus essentiel : la question n'est peut-être pas principalement de se demander comment appeler ces littératures mais de savoir comment appréhender de façon cohérente les littératures francophones, héritières d'un passé, afin de pouvoir les penser au mieux à la fois en synchronie et en diachronie. Cette question est subordonnée à une réflexion indispensable - que chaque enseignantchercheur et chaque participant au marché du livre devrait se poser : quelle est l'importance de la littérature ? Quels enjeux y a-t-il à l'enseigner, à la transmettre ? Certes, ces questions sont banales; pourtant, on s'aperçoit bien vite qu'elles sont rarement posées...

Dans des situations où leur reconnaissance est défaillante, à défaut de pouvoir toucher les institutions, il faut pouvoir transmettre efficacement ces textes francophones au public par l'enseignement - les étudiants, voire les lycéens - ou par les médias; il faut pouvoir les inscrire dans le paysage culturel. Pour cela, il est impératif de dépasser les lectures affectives ou les interprétations au service des revendications particulières. C'est là qu'intervient l'enseignant dont le rôle critique ne consiste pas à décortiquer, classifier, réduire, compresser, de façon stricte et obtuse, mais vise tant bien que mal un objet - les littératures francophones - pour le comprendre, et le donner à comprendre. En sachant que, comme toujours en littérature ou en art, quelque chose débordera, excédera de beaucoup les tentatives pour le circonscrire, le décrire à travers des catégorisations.

Pour les littératures dites du "Sud», l'utilisation, comme le propose Jean-Marc Moura ${ }^{20}$, des outils de la théorie postcoloniale appliqués aux littératures francophones issues des anciennes colonies, peut offrir des perspectives d'approche efficaces. Les outils de la théorie postcoloniale éclairent l'émergence de littératures dites "mineures», les pratiques de décentrement, le positionnement des écrivains immigrés/migrants, l'hybridité... En tant que posture d'interprétation de certains textes et tranche de l'histoire littéraire ${ }^{21}$, cette perspective qui part du conditionnement sociohistorique des poétiques dans leurs relations aux codes et institutions de la métropole, prend acte de l'instabilité, de l'«intranquillité culturelle» vécue par nombre d'écrivains en contexte postcolonial.

20. J.-M. Moura, Littératures francophones et théorie postcoloniale, Presses universitaires de France, 1999.

2I. Un des travers de l'utilisation de la théorie postcoloniale consiste à en faire presque une essence des textes, à définir des auteurs contemporains comme postcoloniaux, à réduire donc certaines œuvres et certains écrivains au postcolonialisme. 
Recherches \& Travaux $-\mathrm{n}^{\circ} 76$

\section{De la diversité factuelle au Divers idéal}

Concernant la diversité littéraire, à l'image de la diversité culturelle, le problème tient donc à la définition des contours et des contenus d'une éventuelle discipline mais aussi de la constitution de corpus cohérents sans lesquels cette discipline ne peut être viable : comment penser ensemble des textes qui ne partagent qu'un standard abstrait de la langue, dont même les pratiques attestent la variété ? La mise en évidence de plusieurs littératures francophones empêche-t-il une cohérence, le partage d'un ou plusieurs paradigmes commun(s) ?

Beaucoup d'études récentes répondent à cela par le partage d'un monde globalisé, métissé, exprimé par une littérature du Divers. La littérature en général et la littérature francophone en particulier - et c'est bien là le cheval de bataille de Michel Le Bris, Nimrod, Abdourahman Waberi... - deviendrait analysable - et, donc, transmissible - comme lieu d'hybridation des identités et non plus comme l'expression d'une nationalité. Dans la pratique, le métissage comme paradigme hypothétique de la modernité ne résume en rien l'ensemble des textes littéraires francophones. À bien y regarder, il ne concerne en fait qu'un nombre limité d'écrivains pour lesquels il convient à chaque fois d'en réévaluer la pertinence. Le Divers glissantien est, au regard des textes, une notion abstraite, difficilement applicable, même aux romans de l'auteur, car trop générale. En fait, l'écrivain se situe le plus souvent (ce n'est pas systématique) au croisement de deux ou trois identités culturelles - rarement plus - qu'il s'efforce de concilier autant que faire se peut. On est assez loin du nomadisme tranquille à travers les cultures du monde ou du Divers que propose Édouard Glissant.

Pour l'écrivain francophone, la participation à un champ littéraire, la nationalité politique et le sentiment identitaire, dont le texte est le premier agent, ne concordent pas. Ces "décalages» sont visibles chez l'écrivain antillais : de nationalité française, il revendique une identité spécifique, en rupture avec le centre, mais il s'associe à ce dernier en y publiant. L'accent mis par les études postcoloniales sur l'hybridité conduit plus heureusement à la relativisation des catégories nationales. Il n'est d'ailleurs par rare d'entendre des écrivains s'élever contre la fâcheuse manie qu'ont les critiques de leur coller des étiquettes. On sait par ailleurs que les questions d'appartenance des écrivains sont souvent cruciales pour les communautés lorsqu'elles ne les montent pas les unes contre les autres.

Ces querelles d'appartenance ou de non-appartenance sont accentuées par les abus de langage : on parle d'appartenance à une littérature, alors qu'en 
fait l'écrivain participe à. Ou encore : s'il appartient, c'est de façon plus ou moins déclarée à un paysage, à un lieu, à une communauté... Cette participation ou appartenance ne repose donc pas toujours sur un patron national : l'identité s'étage du local au transnational. On s'affranchit ainsi d'un enclos national dans la mesure où l'écrivain peut participer à plusieurs littératures, sans pour autant décrier les revendications identitaires. Relativiser la «nationalité» du texte, et donc celle d'un corpus précis, ne veut pas dire que l'on évacue la localité du texte, ni qu'un texte ne peut être l'agent d'une littérature nationale. Cela suppose plutôt que la constitution d'un corpus francophone devrait être attentif à l'identité culturelle - ou à la localité - que la triade auteur-texte-contexte désigne. Je reviendrai plus loin sur les éléments qui rendent possible cette analyse.

Auparavant, nous pourrions, semble-t-il, distinguer deux "niveaux", embrayés l'un sur l'autre, convergents dans le cadre de la littérature française francophone ou de la littérature québécoise francophone, parfois antagonistes pour d'autres écrivains francophones, postcoloniaux notamment :

- le champ littéraire local - lorsquill existe - inséré dans un réseau international accouplé au champ médiatique;

- la participation à un imaginaire socioculturel, symbolique; le sentiment d'appartenance à un lieu, à un monde, c'est-à-dire tout ce qui relève du dialogue fécond du réel avec une poétique productrice d'imaginaires, mais aussi des discours sur une société à partir de jeux portant sur les codes.

\section{L'interaction langue et culture}

Eu égard à cette diversité, pour ne pas dire hétérogénéité, une première façon d'appréhender la francophonie littéraire consisterait à s'intéresser à l'appropriation de la langue française et au discours qui souvent l'accompagne. Dans des contextes de diglossie ou de plurilinguisme, l'étude du bilinguisme, de la bi-langue selon Khatibi, ou encore de la surconscience linguistique au cour de l'interaction entre langue et culture ${ }^{22}$ donnent un accès pertinent à la complexité des énonciations francophones d'écrivains tels que Seyhmus Dagtekin ou Tchicaya U Tam'si. Le travail à l'intérieur d'une langue maternelle mais historiquement imposée persiste aujourd'hui. C'est le cas aussi pour le rapport à la langue qu'entretiennent les auteurs qui ont choisi le français comme langue d'écriture, de la Roumanie à l'Amérique du Sud, en passant par les

22. L. Gauvin, L'Écrivain francophone à la croisée des langues, Karthala, 1997, p. 7-9. L'auteur insiste sur le choix d'une langue comme "acte de langage", "procès littéraire plus important que les procédés mis en jeu». 
Balkans : un tel choix implique un imaginaire de la langue. On peut percevoir cette surconscience comme une spécificité de la francophonie, à toujours bien requalifier, remotiver en fonction des corpus.

Cette surconscience littéraire trouve son origine dans un décalage entre langue et culture : entre une langue et un territoire culturellement autre à la suite d'un choix ou d'une "imposition", c'est-à-dire dans un mouvement de déterritorialisation et de reterritorialisation de la langue et de l'identité. L'écrivain francophone, selon cette perspective, serait un "passeur» ou "médiateur ${ }^{23} \mathrm{de}$ cultures dans une circulation du passé vers le présent puis le futur, de la terre d'origine vers le monde ou inversement, dans la tension de la localité vers l'universel à travers une langue partagée. Pour l'écrivain francophone, le français s'incarne dans un imaginaire culturel, se frotte aux autres langues, s'infléchit en leur présence, se nourrit des autres écritures.

En retour, cette acception de la francophonie littéraire spécifie comme francophones d'éventuelles productions en français d'auteurs basques, occi$\operatorname{tans}^{24}$, bretons... ou encore immigrés qui jugeraient la culture française comme dominante et utiliseraient la langue française pour dire leur identité minorisée. Ceci est relatif à l'histoire de la langue française ayant "conquis» des territoires en métropole avant de s'étendre au delà des frontières. On est donc amené à faire la proposition suivante : si l'écrivain français est majoritairement francophone au sens étymologique, il l'est aussi parfois au sens littéraire : minoritaire contre la pensée dominante, reterritorialisant la langue française pour se dire. Cette dynamique, cependant, ne vaut pas pour tous les auteurs; elle traverse les francophonies comme un mouvement historique, plus fortement marqué en certains endroits qu'en d'autres.

\section{Une perspective comparatiste}

Un second pivot problématique s'intéresserait, lui, aux mémoires et imaginaires culturels francophones à travers des motifs et «figures» mythiques et historiques transversales. Pour aller vite, si les études sur les rapports entre mythes - au sens élargi comprenant les mythes littéraires et artistiques - et littérature abondent dans la littérature française et européenne en général, jusqu'à en avoir saturé le domaine, le terrain est inexploré pour les littératures francophones. Pourtant, les figures historiques et légendaires ne manquent

23. À défaut de trouver un paradigme, Michel Beniamino pense la francophonie littéraire comme frontière, lieux de passage, faisant de l'écrivain un passeur. Voir M. Beniamino, «La francophonie littéraire", Les études littéraires francophones : état des lieux, op. cit., p. 15-24.

24. Pourquoi, à la limite, ne pas se pencher sur l'œuvre bilingue de Max Rouquette? 
pas, et elles présentent l'avantage de véhiculer une mémoire et un imaginaire collectifs dépassant souvent les localités.

Plus largement, les littératures francophones gagneraient à être lues et enseignées selon une perspective comparatiste. Celle-ci n'évincerait pas les "spécialités», mais y aurait recours la spécificité de chaque texte. Il serait ainsi possible d'étudier la disponibilité d'une mémoire, d'un sujet, ainsi que sa singularisation dans les textes d'horizons variés, ainsi que de réfléchir à la circulation des discours, des formes et des imaginaires, c'est-à-dire des pensées. Pour la France, ce comparatisme est l'interface indispensable pour effectuer enfin le dialogue entre textes "français» et autres francophones, cette liaison étant valable pour toute «localité». Il pourrait sans doute contribuer ainsi à une réflexion plus ample sur la redéfinition des corpus et la transmission du patrimoine littéraire.

\section{Pour une histoire littéraire comparée}

Plusieurs critiques - Marc Quaghebeur, Martine Mathieu-Job, Michel Beniamino, Pierre Halen et Jean-Marc Moura pour ne citer qu'eux - ont appelé à une histoire comparée des littératures francophones. À leur façon, Jean-Louis Joubert et Dominique Combe ont déjà posé quelques repères. En conclusion du colloque sur les études francophones, qui s'est tenu à l'université Lille 3, Jean-Marc Moura et Lieven D'Hulst déploraient le manque d'une histoire générale des lettres francophones :

En raison de sa complexité, elle ne peut se construire sur le modèle des histoires littéraires nationales occidentales et doit répondre aux questions de l'élaboration d'historiographies particulières et d'une histoire mettant celles-ci en relation sans les dissoudre dans les mirages d'une généralité sans rivages. L'entreprise est risquée ${ }^{25}$.

De même, le centre de recherche "Textes et Francophonies » à l'université de Cergy-Pontoise se penche actuellement sur la mise en place d'une telle histoir ${ }^{26}$, en travaillant à édition de deux dictionnaires de classiques francophones. C'est dans cette perspective qu'il faudra inscrire des recherches sur l'histoire de la littérature française dans son rapport avec les influences extérieures ${ }^{27}$, afin de voir comment cet espace est traversé d'«éléments transnationaux». Avec la

25. L. D'Hulst, J.-M. Moura, Les études littéraires francophones : état des lieux, op. cit.

26. C. Chaulet-Achour, Convergences Francophones, Encrage-Université de Cergy-Pontoise, Amiens, 2006.

27. Pour quelques pistes, voir C. Chaulet-Achour, "Qu'entend-on par "francophonies littéraires" ", Convergences francophones, op. cit. 
notion de «scène», Judith Schlanger invite à mieux comprendre le présent littéraire en le regardant comme aboutissement d'une histoire propre : l'actualité littéraire est plurielle parce que les centres ou les scènes dans lesquelles elle se joue se trouvent à l'issue d'une histoire ou d'une aventure singulière :

[La littérature] apparaît [...] comme une activité extrêmement volontaire qui se joue dans de multiples scènes locales, en fonction des situations concrètes, à travers des milieux chargés de désirs, de contentieux, de conscience d'obstacles et d'objectifs. Et ce sont ces situations de parole (situations historiques, politiques, sociologiques, idéologiques, idéales ou traditionnelles) qui peuvent devenir un objet d'étude propre ${ }^{28}$.

Lobjectif majeur de cette histoire ? Amener une compréhension des phénomènes, des "scénarios locaux » hérités de l'Histoire, en vue d'une transmission d'un patrimoine pluriel, mouvant, métissé. Penser le texte littéraire dans une situation au confluent de culture(s), sociétés, nations et d'une histoire plurielle permet de conférer un semblant de cohérence aux francophonies littéraires en saisissant ce qui est partagé ou "différencié». Comparée, cette histoire l'est nécessairement dans la mesure où chacune des francophonies est confrontée à une ou des "historicités toujours marquées par la dépendance et l'interdépendance évidentes, voire par un destin subi ${ }^{29}$ ".

Un second objectif nous ramène à ce qui a été évoqué plus haut : faire évoluer le patrimoine littéraire en repensant les corpus traditionnels. À ceux-ci seraient intégrés de nouveaux textes, ouvrant ce patrimoine à d'autres imaginaires, pendant que d'autres œuvres seraient replacées dans la mémoire, c'est-à-dire remises en évidence.

Malheureusement, au niveau le plus structuré et matériel, celui du système éditorial et de diffusion, on s'aperçoit bien vite de l'absence de circulation réelle des textes littéraires : les productions sont cantonnées aux régions ou aux pays et sont tributaires des lois aliénantes du marché. Un texte acadien, exception faite d'un coup de projecteur exotique comme celui qui a été braqué sur Antonine Maillet, n'aura presque aucune chance de se retrouver sur les tables des libraires parisiens. La distance géographique n'est pas en cause : les œuvres de la plupart des écrivains suisses ${ }^{30}$, inconnus de ce côté-ci des Alpes, reconnus de l'autre, sont difficilement accessibles en France. En outre, les textes publiés dans des maisons d'édition sans pouvoir dans le système échappent à la diffusion. Les écrivains francophones connus

28. Ibid., p. 93.

29. M. Quaghebeur, "Et si nous parlions enfin des francophonies culturelles ?", Où va la francophonie au début du troisième millénaire?, G. Dotoli (dir.), Fasano-Paris, Schena - Presses universitaires de Paris-Sorbonne, 2005, p. 69.

30. Citons A.-L. Grobéty, J. Chessex, A. Perrier, P. Chappuis, C. Bille... Et, pourquoi pas, y compris les «classiques» G. Haldas, G. Roud, M. Chappaz... 
en France ont en majorité été publiés dans des grandes maisons d'édition françaises comme Gallimard, Le Seuil, Grasset. Des éditeurs plus modestes assurent néanmoins une diffusion plus ou moins "en sourdine", par exemple les Éditions de l'Aube, implantées à la Tour d'Aigues ou Le Serpent à Plumes. De ce côté, il n'y a donc pas de vrai ensemble francophone ${ }^{31}$, et cela induit un problème de fond, très concret : comment penser des littératures, transmettre des textes, ouvrir les corpus lorsque même les textes «majeurs» étrangers en (une) langue française sont mal diffusés?

Pour revenir au débat francophone, nous pouvons espérer que, grâce à l'histoire littéraire préconisée, seront évitées les altercations entre profrancophones et anti-francophones, car elle fera prendre un recul critique à l'égard de tout ce que la langue française a cristallisé32. Rien de mieux que la compréhension des causes du présent pour se déprendre des réactions impulsives. Une telle entreprise permettrait d'échapper au procès d'intention intenté à la francophonie littéraire par une lecture soucieuse de la complexité des faits littéraires et dont l'ossature reposera sur une pratique critique de l'histoire de la langue à travers les espaces. Ce n'est qu'en pensant le texte avec l'histoire, dans l'histoire que l'on remédiera aux différends.

Une telle histoire en manuels, possible uniquement par le concours d'un grand nombre d'enseignants, d'équipe de chercheurs et d'écrivain(e)s, n'a donc d'abord pour vocation ni de résoudre l'équation francophone, ni de réaliser la somme de toutes les œuvres, mais d'expliciter la circulation littéraire, la connexion entre les textes de cultures différentes. La synthèse s'effectuerait à plusieurs niveaux à articuler impérativement :

- Un savoir extra-littéraire historique, sociopolitique et culturel : c'est un passage tout simplement indispensable pour comprendre le texte littéraire dans sa richesse.

- Une histoire de l'esthétique littéraire : non seulement formatrice pour comprendre l'imaginaire, la "poétique» a également d'évidentes justifications et implications culturelles, idéologiques et esthétiques.

- Une sociologie de la littérature : au niveau des "champs littéraires» ou, plus exactement les systèmes éditoriaux, commerciaux et institutionnels qui

3I. P. Halen, "Le système littéraire francophone», Les études littéraires francophones : état des lieux, Actes du colloque 2-4 mai 2002, Éditions du Conseil scientifique de l'université Charles-de-Gaulle - Lille III, Villeneuve-d'Ascq, 2003, p. 4I. P. Halen va jusqu'à se demander si cet objet - le domaine des littératures francophones - existe.

32. Aux «adversaires» de la francophonie littéraire, on peut faire remarque que tous les enseignants de littérature francophone ne sont pas des néocolonialistes, bien au contraire : c'est par eux que pourra venir la fin de la querelle. 
régissent le cours des faits littéraires. Pierre Halen a élaboré le schéma d'un système littéraire pertinent à bien des égards, et qui présente l'avantage considérable de ne pas se cantonner à un seul "champ" ni de les superposer ${ }^{33}$. Dans ce système, on peut découper des scènes littéraires ${ }^{34}$ dont il a déjà été question plus haut. Ces scènes s'instaurent dans des contextes sociopolitiques, culturels, physiques, ou encore linguistiques : elles sont précisément ce fameux lieu d'où parle l'écrivain au croisement du politique, de la société et de la culture.

Il ne s'agit pas de servir par là une autre idéologie, ni d'assouvir des désirs encyclopédiques, mais de proposer des mises en perspective pour agir sur les corpus :

Car il s'agit, ici comme ailleurs, de parvenir, dans notre langue et dans l'état actuel de cette langue, à dire des Histoires et des spécificités auxquelles, à de nombreux égards et à certains moments, les mots tels que la norme française les a définis, résistent. [...] C'est là, bien évidemment, la chance qu'offrent les francophonies à notre langue - et donc à la France. À condition de s'ouvrir réellement à la question du pluriel et de la perpétuelle tension qu'il instaure ${ }^{35}$.

L'étude des littératures francophones doit avoir conscience qu' elle découpe, en fonction d'une problématique locale ou transnationale, un corpus situé dans un ensemble excédant toute tentative pour le circonscrire; et que, par ce biais, elle opère le choix d'une perspective qui ne constitue pas la seule manière d'aborder la francophonie littéraire.

Pour conclure, je crois que, sans se soucier seulement de l'avenir de la langue française, on pourrait s'inquiéter de celui de la littérature en général. Je souscris aux propos d'Alain Mabanckou, qui ne sont malheureusement pas ce que l'on aura retenu du manifeste :

[...] l'inévitable interrogation qui ne cessera de nous hanter, de nous obnubiler tant que nous ne nous serons pas prononcés : qu’apportons-nous au monde, ou que devrions-nous apporter au monde, nous autres écrivains qui avons en

33. Pour ce faire, il passe par une perspective d'abord sociocritique tout en récusant la notion de "champ" pour prôner celle de «système», plus propice à la complexité réelle des relations entre littératures. L'unité est assurée par la relation entre la force centrifuge du centre parisien, toujours très forte, bien que concurrencée par d'autres pôles comme celui de Montréal.

34. J. Schlanger, "Les scènes littéraires", Où est la littérature mondiale, Chr. Pradeau et T. Samoyault (dir.), Presses universitaires de Vincennes, Saint-Denis, p. 85-97. Locales, elles sont des «situations de parole».

35. Ibid., p. 73. 


\section{LE DÉBAT FRANCOPHONE}

partage la langue française ? La réponse à cette question traduira notre posture à venir. Y répondre, c'est entamer l'édification d'une forteresse ${ }^{36}$.

Toute approche littéraire, quelle que soit la démarche critique qu'elle emprunte, dès lors qu'elle se posera cette question, gardera la cap sans se perdre dans des bavardages abscons, et saura dialoguer avec tout discours littéraire et métalittéraire. Derrière le fracas des bordées en francophonie(s), la question posée en creux dans le débat n'en reste pas moins beaucoup plus cruciale, autrement aiguë : celle de la place de la littérature aujourd'hui et pour demain. C'est une des questions universelles pouvant unir «Nord» et "Suds», ces fictions que sont l'Occident et l'Orient, les anciennes colonies et les métropoles, les écrivains et les critiques, les lecteurs ordinaires et les enseignants.

36. A. Mabanckou, op. cit., p. 62. 